\title{
pET expression vector customized for efficient seamless cloning
}

\author{
Dragana Dobrijevic $^{1}$, Lily A Nematollahi ${ }^{2}$, Helen C Hailes $^{3}$ (D) \& John M Ward ${ }^{\star, 1}$ (D) \\ ${ }^{1}$ Department of Biochemical Engineering, University College London, Gower Street, London, WC1E 6BT, UK; ${ }^{2}$ Synthace Ltd, The Westworks, 195 Wood Lane, London, W12 \\ 7FQ, UK; ${ }^{3}$ Department of Chemistry, University College London, 20 Gordon Street, London, WC1H 0AJ, UK; *Author for correspondence: j.ward@ucl.ac.uk
}

BioTechniques 69: 00-00 (June 2020) 10.2144/btn-2020-0101

First draft submitted: 13 July 2020; Accepted for publication: 8 September 2020; Published online: 30 October 2020

\section{ABSTRACT}

Here we present a modification of the widely used pET29 expression vector for use in rapid and straightforward parallel cloning via a gene replacement and Golden Gate strategy. The modification can be applied to other expression vectors for Gram-negative bacteria. We have used the modified vectors to clone large numbers of bacterial natural enzyme variants from genomic and metagenomic sources for applications in biocatalysis.

\section{METHOD SUMMARY}

The pET29 vector was modified to contain, instead of the multiple cloning site, a negative selection marker (sacB) flanked by Type Ils restriction sites. One-pot restriction-ligation reaction, transformation into Escherichia coli and plating on selective media yields $>98 \%$ recombinant plasmids containing the gene of interest fused to the His6-tag at the C-terminus or, if required, genes that do not contain any vector-encoded sequences.

\section{KEYWORDS:}

expression in E. coli • Golden Gate • pET vectors • sacB • seamless cloning

Rapid advances in next-generation sequencing technologies have generated vast amounts of genomic sequence data that represent a rich source for bioprospecting for enzymes for industrial biotechnology applications. In order to speed up enzyme discovery and functional analysis, tools and protocols for high-throughput cloning are necessary. A variety of cloning strategies have been developed for creating protein expression constructs for structural and functional studies [1]. The Golden Gate cloning strategy has been shown to be incredibly powerful in cloning DNA fragments with high efficiency [2-5]. It relies on the use of Type IIs restriction enzymes that cleave DNA outside of their recognition site, providing unique cohesive ends that enable directional and seamless cloning of the gene of interest. The Golden Gate protocol allows for convenient and rapid cloning in a single-tube, one-step coupling restriction digestion and ligation [2].

Vectors from the pET System (Novagen) are a popular choice for the heterologous expression of genes in Escherichia coli under control of the T7lac promoter for applications that require high protein yields. Here we present the modification of one of these vectors, $p E T 29 a(+)$, for use with the Golden Gate cloning strategy. Two vectors were generated and designated pET29:SacB-Bsal and pET29:SacB-Sapl. Both contain a negative selection marker in place of the multiple cloning site of pET29: the Bacillus subtilis sacB gene, expressed from its native promoter and flanked by Bsal or Sapl restriction sites (Figure 1A).

The sac $B$ gene from $B$. subtilis encodes levansucrase, an enzyme secreted in the culture medium after induction by sucrose. Levansucrase catalyzes the hydrolysis of sucrose and synthesis of the branched fructose polymer known as levan. Expression of sac $B$ in $E$. coli and other Gram-negative bacteria is lethal in the presence of sucrose, probably due to the accumulation of levans in the periplasm [6-8]. In $B$. subtilis, the upstream region of the $\operatorname{sac} B$ gene contains the promoter and the regulatory sequence sacR which, when cloned together with $\operatorname{sacB}$, promote its efficient expression in $E$. coli [9]. We thus amplified the 1903-bp region from $B$. subtilis containing 445 bp of upstream regulatory sequences, the $s a c B$ gene and the terminator sequence, and inserted it in the pET29, where it replaced the multiple cloning site in the opposite orientation to the T7lac promoter [10]. We found that this orientation resulted in efficient sacB expression and counterselection.

All molecular biology reagents were obtained from New England Biolabs and used following the manufacturer's protocols, unless stated otherwise. All PCR reactions were performed with the Phusion ${ }^{\circledR}$ High-Fidelity PCR Master Mix with HF Buffer. PCR products were gel-purified or PCR-purified with Monarch ${ }^{\circledR}$ PCR \& DNA Cleanup Kit. All ligation reactions were performed with the high-concentration T4 DNA ligase. Restriction digestion protocols were performed with restriction enzymes: Ndel, Xhol, Dpnl, Sapl and Bsal-HFv2. QiaQuick Plasmid Kit (Qiagen) was used for plasmid DNA preparation. All primers were synthesized by Eurofins Genomics and are listed in Table 1. 
(A)

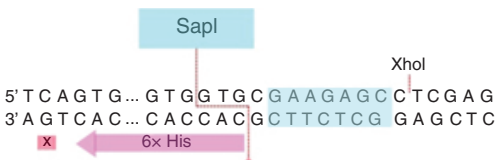

IX

$$
\mathrm{X}
$$

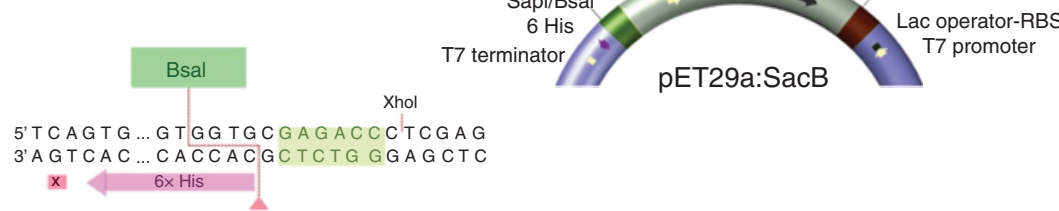

5'G A CG CT C T T C C C A T A T GTATA

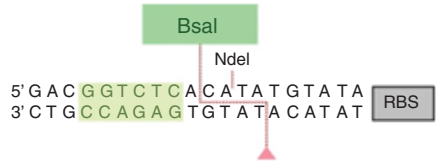

(B)

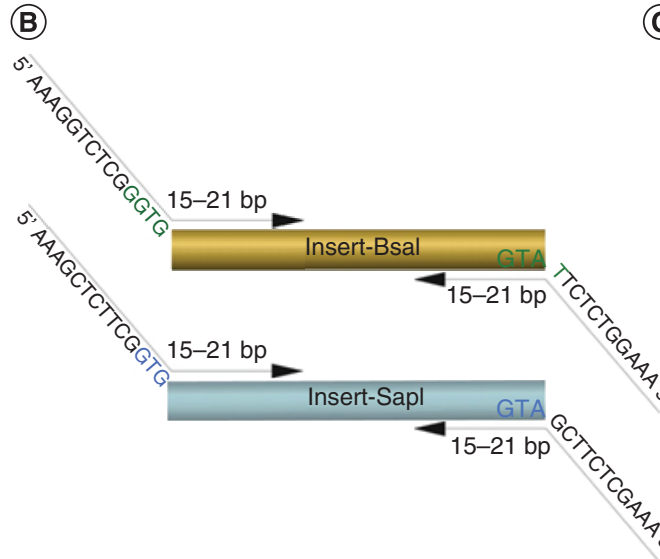

(C)

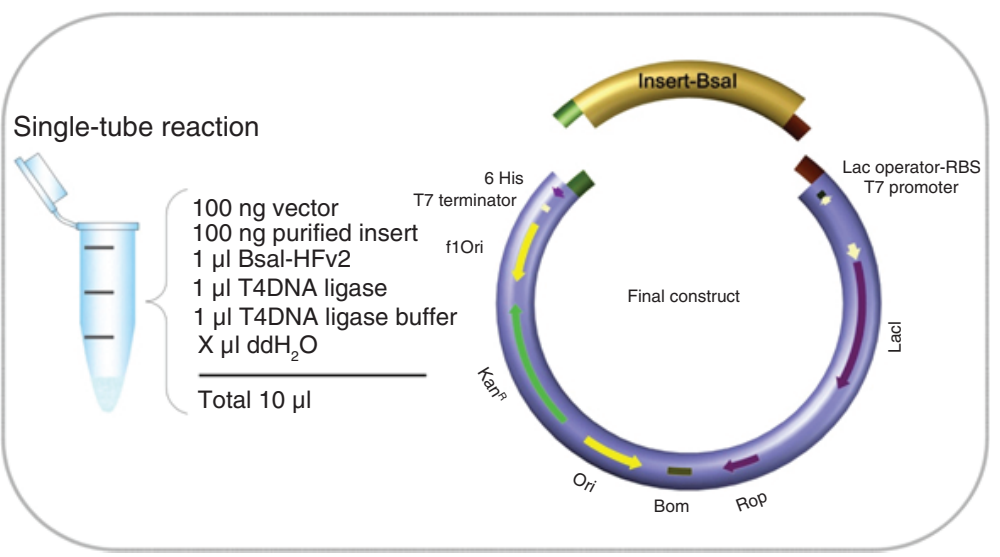

Figure 1. One-pot cloning reaction with modified pET vector and a single insert. (A) Bsal and Sapl restriction sites allow excision of sacB and result in 4-base and 3-base overhangs, respectively, that direct the assembly. (B) Recognition sequences for Sapl and Bsal are added via the PCR to the gene of interest. Complementary overhangs in the insert that direct the assembly are in bold, green and blue. (C) In the single-tube reaction, the sacB in the destination vector is replaced with the gene of interest so that the ATG within the Ndel site (CATATG) is used as a start codon, putting the gene of interest in-frame with vector-encoded C-terminal His6-tag. In the final construct no amino acids are present between the protein of interest and the His6-tag.

\begin{tabular}{|c|c|}
\hline Primer & Sequence $\left(5^{\prime}-3^{\prime}\right)$ \\
\hline pET29-Bsal-F & AAACATATGTATATCTCCTTCTTAAAGTTAAACAAAATTATTTCTAG \\
\hline pET29-Bsal-R & AAAACTCGAGGGTCTCGCACCACCACCACCACCACTGAG \\
\hline sacB-Bsal-F & AAACATATGTGAGACCGTCAATGCCAATAGGATATCGGCATTTTC \\
\hline sacB-Bsal-R & AAAACTCGAGACATATACCTGCCGTTCACTATTATTTAGTG \\
\hline P3-F & P-GCTTCCTCGCTCACTGACTCGCTG \\
\hline P3-R & P-GGAAGTGCGCCTGATGCGGTATTTTCTCCTTACG \\
\hline pET29-Sapl-F & AAACATATGTATATCTCCTTCTTAAAGTTAAACAAAATTATTTCTAG \\
\hline pET29-SapI-R & AAAACTCGAGGCTCTTCGCACCACCACCACCACCACTGAG \\
\hline sacB-Sapl-F & AAACATATGGGAAGAGCGTCAATGCCAATAGGATATCGGCATTTTC \\
\hline sacB-Sapl-R & AAAACTCGAGACATATACCTGCCGTTCACTATTATTTTAGTG \\
\hline
\end{tabular}

Ndel and Xhol restriction sites are in bold; Bsal and Sapl restriction sites are underlined. Additional nucleotides introduced during the cloning procedure are in gray.

E. coli Nova Blue cells from Novagen were made chemically competent using the standard calcium chloride protocol. The working concentration for kanamycin was $50 \mu \mathrm{g} / \mathrm{ml}$.

The $B$. subtilis sacB coding sequence, complete with its signal peptide and regulatory sequences (GenBank CP051860.1: 1746670$1748572,1903 \mathrm{bp}$ ), was previously amplified from strain 168 genomic DNA and cloned into a customized vector. To construct pET29:SacB$B$ sal, sacB and pET29a(+) were first PCR amplified using the primers sacB-Bsal-F/sacB-Bsal-R and pET29-Bsal-F/pET29-Bsal-R, re- 
spectively. PCR fragments were then digested with Ndel and Xhol restriction enzymes and ligated to form the final vector. To construct pET29:SacB-Bsal, the Sapl restriction site was deleted in the pET29a(+) by changing the sequence from GCTCTTC to GCACTTC. $5^{\prime}$ phosphorylated primers P3-F and P3-R were used to do this in a routine PCR reaction with 20 cycles. The PCR reaction product was digested with $D p n$ to remove the methylated template vector and thus reduce the background. PCR products were gel-purified, ligated to form circular vectors and transformed into chemically competent $E$. coli Nova Blue cells. The vector with deleted Sapl restriction site was PCR amplified using the primers pET29-Sapl-F and pET29-Sapl-R. The PCR product was digested with Ndel and Xhol and ligated to sacB that was amplified with sacB-Sapl-F and sacB-Sapl-R and digested with the same restriction enzymes.

A functional $s a c B$ gene is a prerequisite for efficient counter selection; therefore after ligation and transformation, individual colonies were tested for their growth on Luria-Bertani medium supplemented with kanamycin, in the presence or the absence of $10 \%$ sucrose. Vectors were isolated from transformants that were resistant to kanamycin and sensitive to sucrose. Both constructed vectors, $\mathrm{pET} 29$ :SacBBsal and pET29:SacB-Sapl, were further verified by DNA sequencing and their functionality was initially tested by cloning and expression of eGFP in E.coli BL21 (DE3) (data not shown).

To clone the genes of interest in the modified vectors, primers generally contained a minimum of 15 (preferably 18-21) nucleotides complementary to the sequence of interest with, when possible, a GC content of about $50 \%$. The following overhangs were added to the primers: Forward 5'-AAAGGTCTCTTATG-3' and Reverse 5'-AAAGGTCTCGGGTG-3' for cloning in pET29:SacB-Bsal; and Forward 5'AAAGCTCTTCGATG-3' and Reverse 5'- AAAGCTCTTCGGTG-3' for cloning with pET29:SacB-Sapl. Overhangs contained restriction sites and were flanked by three 'spacer' nucleotides at the $5^{\prime}$ end to allow for efficient digestion (Figure 1B). In our case, the choice of the three- and four-base overhangs was restricted by the vector sequence, whereas in multipart assemblies junctions can often be arbitrarily chosen [11,12]. To avoid the vector-encoded C-terminal Hisx6 fusion, a translation stop codon could be included in the insert via the primer. Genes were amplified using the standard PCR procedure and gel-purified before being used in the one-pot cloning reaction.

The one-pot cloning reaction mixture consisted of $100 \mathrm{ng}$ vector, $100 \mathrm{ng}$ amplified DNA fragments, $1 \mu \mathrm{l}$ restriction enzyme, $1 \mu \mathrm{l}$ T4 DNA ligase and $1 \mu \mathrm{l} 10 \times$ T4 DNA ligase buffer in a total reaction volume of $10 \mu \mathrm{l}$ (Figure $1 \mathrm{C}$ ). The mixture was incubated at $37^{\circ} \mathrm{C}$ for $30 \mathrm{~min}$. $2 \mu \mathrm{l}$ of each reaction was added to $20 \mu \mathrm{l}$ of Nova Blue $E$. coli chemically competent cells and incubated on ice for 30 min. The cells were heat shocked at $42^{\circ} \mathrm{C}$ for $60 \mathrm{~s}$, chilled on ice for $5 \mathrm{~min}$, then recovered in $200 \mu \mathrm{l}$ of SOC for $1 \mathrm{~h}$ at $37^{\circ} \mathrm{C}$. Cells were plated on Luria-Bertani agar supplemented with kanamycin and $10 \% \mathrm{v} / \mathrm{v}$ sucrose (sterile sucrose solution was prepared by filtering and added to warm Luria-Bertani agar before pouring into plates). Typically $>98 \%$ of clones positive for the desired recombinant vector were obtained after transformation. This high efficiency eliminated the need for colony screening and allowed for a single colony per construct to be picked and verified by DNA sequencing.

The method described has been extensively used in our laboratory to construct and expand our biocatalysis enzyme toolbox with a wide range of enzymes. It does not require very long primers or commercial kits. Cloning is directional and the protocol has proved to be fast, reliable and efficient; in a great majority of the clones, only the desired product is present. One-pot restriction digestion/ligation is carried out in a short time (30 mins) and the ligated DNA is immediately transformed. Expression-ready clones are available the next day after growth of the transformed cells. To date, using the adapted vectors, we have effectively cloned and expressed in $E$. coli hundreds of genes with diverse origins (from metagenomic DNA and individual genomes) and complexity, with GC content ranging from 35 to $73 \%$ and gene lengths between 0.3 and $2.5 \mathrm{~kb}$. Examples of panels of recombinant enzymes constructed in this way include enzyme classes such as transaminases from a drain metagenome [13], ene-reductases also from a drain metagenome [14] and epoxide hydrolases mined from sequenced bacterial genomes [15], demonstrating the versatility of the protocol.

\section{Author contributions}

D Dobrijevic and L Nematollahi conceived the experiments; D Dobrijevic designed and performed the experiments and analyzed the data; D Dobrijevic and L Nematollahi wrote the manuscript. All authors edited and approved the final manuscript. Funding was from grants to D Dobrijevic, J Ward and H Hailes.

\section{Acknowledgments}

The authors acknowledge the UKRI Research Councils BBSRC and EPSRC for financial support to D Dobrijevic and J Ward.

\section{Financial \& competing interests disclosure}

D Dobrijevic is supported by the BBSRC grant BB/L007444/1 and the EPSRC grant EP/S024883/1. Other support for J Ward and H Hailes is from the BBSRC grant BB/N01877X/1. The authors have no other relevant affiliations or financial involvement with any organization or entity with a financial interest in or financial conflict with the subject matter or materials discussed in the manuscript apart from those disclosed.

No writing assistance was utilized in the production of this manuscript. 


\section{Open access}

This article is distributed under the terms of the Creative Commons Attribution License 4.0 which permits any use, distribution, and reproduction in any medium, provided the original author(s) and the source are credited. To view a copy of the license, visit http://creativecommons.org/licenses/by/4.0/.

\section{References}

Papers of special note have been highlighted as: $\bullet \bullet$ of considerable interest

1. Celie PHN, Parret AHA, Perrakis A. Recombinant cloning strategies for protein expression. Curr. Opin. Struct. Biol. 38, 145-154 (2016).

2. Engler C, Kandzia R, Marillonnet S. A one pot, one step, precision cloning method with high throughput capability. PLoS ONE 3(11), e3647 (2008).

-. First description of the Golden Gate cloning strategy that allows highly efficient directional assembly of multiple DNA fragments.

3. Engler C, Gruetzner R, Kandzia R, Marillonnet S. Golden Gate shuffling: a one-pot DNA shuffling method based on Type Ils restriction enzymes. PLoS ONE 4(5), e5553 (2009).

4. Weber E, Engler C, Gruetzner R, Werner S, Marillonnet S. A modular cloning system for standardized assembly of multigene constructs. PLoS ONE 6(2), e16765 (2011).

-. Modular cloning system based on Golden Gate that allows assembly of multigene constructs from sets of pre-made standardized genetic modules.

5. Iverson SV, Haddock TL, Beal J, Densmore DM. CIDAR MoClo: improved MoClo assembly standard and new E. coli part library enable rapid combinatorial design for synthetic and traditional biology. ACS Synth. Biol. 5(1), 99-103 (2016).

-. Publicly available library of standardized DNA parts and assembly protocols for use in $E$. coli based on Golden Gate.

6. Ried JL, Collmer A. An nptl-sacB-sacR cartridge for constructing directed, unmarked mutations in Gram-negative bacteria by marker exchange-eviction mutagenesis. Gene 57(2-3), 239-246 (1987)

7. Steinmetz M, Le Coq D, Aymerich S, Gonzy-Tréboul G, Gay P. The DNA sequence of the gene for the secreted Bacillus subtilis enzyme levansucrase and its genetic control sites. Mol. Gen. Genet. 200(2), 220-228 (1985)

8. Gay P, Le Coq D, Steinmetz M, Berkelman T, Kado Cl. Positive selection procedure for entrapment of insertion sequence elements in Gram-negative bacteria. J. Bacteriol. 164(2), 918-921 (1985).

9. Gay P, Le Coq D, Steinmetz M, Ferrari E, Hoch JA. Cloning structural gene sacB, which codes for exoenzyme levansucrase of Bacillus subtilis: expression of the gene in Escherichia coli. J. Bacteriol. 153(3), 1424-1431 (1983).

10. Wu SS, Kaiser D. Markerless deletions of pil genes in Myxococcus xanthus generated by counterselection with the Bacillus subtilis sacB gene. J. Bacteriol. 178(19), 5817-5821 (1996).

11. Vladimir P, Ong J, Kucera R et al. Optimization of Golden Gate assembly through application of ligation sequence-dependent fidelity and bias profiling. bioRxiv. 322297 (2018).

12. Potapov V, Ong JL, Langhorst BW et al. A single-molecule sequencing assay for the comprehensive profiling of T4 DNA ligase fidelity and bias during DNA end-joining. Nucleic Acids Res. 46(13), e79 (2018).

13. Leipold L, Dobrijevic D, Jeffries JWE et al.The identification and use of robust transaminases from a domestic drain metagenome. Green Chem. 21(1), 75-86 (2019).

14. Dobrijevic D, Benhamou L, Aliev AE et al. Metagenomic ene-reductases for the bioreduction of sterically challenging enones. RSC Adv. 9(63), 36608-36614 (2019).

15. Stojanovski G, Dobrijevic D, Hailes HC, Ward JM. Identification and catalytic properties of new epoxide hydrolases from the genomic data of soil bacteria. Enzyme Microb. Technol. 139, $109592(2020)$ 